\title{
Vestibular migraine - an underdiagnosed cause of vertigo. Diagnosis and treatment
}

\author{
Magdalena Nowaczewska ${ }^{1,2}$ \\ ${ }^{1}$ Department of Otolaryngology, Head and Neck Surgery, and Laryngological Oncology, Faculty of Medicine, Ludwik Rydygier \\ Collegium Medicum in Bydgoszcz, Nicolaus Copernicus University, Bydgoszcz, Poland \\ ${ }^{2}$ Athleticomed - Pain \& Sport Injury Centre with Headache \& Migraine Treatment Division, Bydgoszcz, Poland
}

\begin{abstract}
Introduction. Migraine and vertigo are two common conditions. The main disorder connecting both these entities is vestibular migraine (VM).

State of the art. VM may affect 1-3\% of the general population. It is a disabling disease of recurrent attacks of vestibular symptoms accompanied by migraine features which occur in patients with a current or previous history of migraine. The episodes can last minutes, hours or even days, and may occur without any concurrent headache, which can prompt misdiagnosis. VM often begins several years after a typical migraine, and the delay between onset of headache and vertigo may be long. The diagnosis is based on the patient's clinical history and can be challenging due to the lack of an established confirmatory diagnostic test or biomarkers. The mechanism of vestibular migraine remains unclear and is still under investigation, but it seems to be an interaction between trigeminal and vestibular systems. Due to the lack of specific trials, treatment recommendations are based on migraine guidelines. Several drugs seem to be effective, although there have been few randomised controlled trials in this area. Regardless of the published strict and detailed diagnostic criteria, this condition remains little known, and as a consequence is underdiagnosed and undertreated.
\end{abstract}

Clinical implications. Efforts should be made to educate medical communities and patients about this disease and to encourage neurologists and ENT specialists to cooperate. Every patient with vertigo of unknown origin should be directly asked about a past or present history of migraine, or migraine symptoms experienced during their vertigo episodes.

Future directions. There is a growing need for studies regarding the pathophysiology of VM as well as randomised trials to establish clear treatment recommendations and to improve management of this surprisingly common disorder.

Key words: migraine, vestibular migraine, headache, dizziness, vertigo, treatment

(Neurol Neurochir Pol 2020; 54 (2): 106-115)

\section{Introduction}

Migraine and vertigo are two very prevalent conditions in the general population [1]. Nevertheless, a link between those two disorders exists: vertigo is $2-3$ times more common in migraine patients, especially migraine with aura patients, than in the general population (ranging from 30 to $50 \%$ ) [2, 3]. In addition, vertigo is reported to coexist with other migraine symptoms during almost half of severe migraine attacks [3].
It is also worth noting that vertigo can be one of the aura symptoms in migraine with brainstem aura, as well as a part of benign paroxysmal vertigo in children [4].

However, the main disorder which connects vertigo with headache is vestibular migraine (VM) (previously known as migrainous vertigo or migraine-associated vertigo). $\mathrm{VM}$ is one of the most common neurological disorders causing vertigo and dizziness [5], but despite its high prevalence and published diagnostic criteria, it remains surprisingly little known, and

Address for correspondence: Magdalena Nowaczewska, Department of Otolaryngology, University Hospital no 1, 9 Marii Skłodowskiej-Curie St.,

85-090, Bydgoszcz, Poland, e-mail: m.nowaczewska@cm.umk.pl 
thus underdiagnosed and undertreated [6]. The cause of this diagnostic challenge may be the wide spectrum of symptoms, the absence of headache in almost half of the attacks, as well as poor knowledge of diagnostic criteria and a lack of cooperation between the neurological and otolaryngological communities.

\section{Epidemiology and demographic factors}

Vestibular migraine affects approximately $1-3 \%$ of the general population and up to $30 \%$ of patients in specialised vertigo or headache centres $[1,7]$. Moreover, benign paroxysmal vertigo of childhood is known to be an early manifestation of migraine. This has a $3 \%$ prevalence in children between the ages of 6 and 12 [8]. In one study, VM was diagnosed in $10.3 \%$ of migraineurs, while another determined its prevalence to be $21 \%$ in the migraine population $[9,10]$. Chia et al. [11] reviewed 208 patients with benign recurrent vertigo diagnosed in a neuro-otology clinic, and found that $87 \%$ of them met ICHD criteria for migraine ( $62 \%$ with aura), and that among migraine patients with vertigo and migraine $70 \%$ met the VM criteria, which is a surprisingly high amount. Indeed, VM patients significantly more often experience migraine with aura, and vestibular symptoms are twice as likely to be reported in patients with migraine with aura compared to individuals without aura $[2,3]$. Plus, according to Martinez et al. [12], almost $40 \%$ of patients with VM meet criteria for chronic migraine.

Importantly, the presence of migraine is extremely high in patients with recurrent unclassified vertigo (ranges from $60-80 \%$ ) [11]. VM is more prevalent in females, with a gender ratio of from 1.5 up to 5.1 [13-15]. The average age at onset of VM is $38-50$ [15-17]. It is worth noting that migraine headache precedes the onset of vestibular attacks, where the age at onset of migraine is $23-32$ years, while the average age at onset of vertigo is $34-38$ years $[15,18,19]$. Moreover, mostly in postmenopausal women, typical migraine attacks may be replaced by vestibular episodes [20]. Interestingly, the time gap between migraine and vestibular symptoms onset may be long, as patients can be headache-free for years before the vestibular symptoms starts [21]. The mean delay between the onset of headache and vertigo is 8-14 years [21, 22]. The majority of VM patients suffer from episodic migraine, and one in four of them have chronic migraine with medication overuse headache [15]. Most VM patients report a family history of the disorder. A history of migraine has been found in $50.8-70.2 \%$ of cases [16]. Notably, some patients also mention a family history of vertigo of no clear origin, and many of them refer to migraine precursors, especially motion sickness during childhood as well as cyclic vomiting, torticollis and/or episodic abdominal pain $[8,16,23]$.

Some authors have suggested that vestibular symptoms in paediatric patients may predispose to the development of VM as adults [18]. One study revealed that up to $78 \%$ of VM patients had experienced carsickness in their lifetime [24]. Other prevalent comorbidities in VM patients are mood disorders including anxiety, depression and sleep disturbance $[7,15,25]$. One study demonstrated that age under 40 , female sex, mood disorders, and prior head trauma were all connected with significantly increased odds of developing VM [7]. VM is one of the most common precipitants of persistent postural perceptual dizziness, a newly defined disorder characterised by persistent dizziness and perceived instability, which is exacerbated in busy visual environments and in an upright position [26]. Approximately $40 \%$ of patients with VM have reported missing work because of their symptoms, which demonstrates the impact of this disease on daily activities and quality of life [1].

VM is an underdiagnosed disorder: a study in a tertiary vertigo centre demonstrated that although VM was finally diagnosed in $20.2 \%$ of patients, it was suspected by the referring doctor in fewer than $2 \%$ of patients [6]. Another study showed that only $20 \%$ of VM patients were correctly diagnosed after visiting a doctor [1]. Similarly, Formaister et al. [7] reported that only $10 \%$ of subjects meeting the criteria for VM were told that migraine was the cause of their dizziness.

Misdiagnosis increases when vestibular symptoms are not associated with headaches and half of the patients are supposed to have Meniere's Disease [15]. A survey among American neurologists and otorhinolaryngologists (ENT) revealed that a neurologist would diagnose VM in vertigo and headache patients more often than an ENT specialist. This finding is alarming because 19\% of ENT specialists and 14.5\% of neurologists have declared that they had never treated a patient with VM before [27].

Our experience is that more patients with VM are found in otolaryngological than in neurological departments, and that to set up a VM diagnosis a neurological consultation must be performed, as most ENT specialists are unfamiliar with migraine diagnostic criteria.

\section{Pathophysiology of VM}

Although the pathogenesis of VM is still unclear, genetic, inflammatory and neurochemical mechanisms have been proposed, mostly based on migraine pathophysiology, as migraine interacts with the vestibular system at many different levels [28-30]. It seems that both the central and peripheral vestibular systems are associated with its pathogenesis.

First of all, many studies have highlighted the overlap between vestibular and migraine pathways, as the caudal parabrachial nucleus receives both afferent peripheral trigeminal nociceptive and vestibular input, and also the trigeminal nerve affects the inner ear by cochlear vasculature innervations [31]. So, the cause of VM may be direct central activation of vestibular centres by the trigeminovascular system together with its effects on the inner ear [32]. It seems that vestibular symptoms come from the vestibular nuclei, which are simultaneously suppressed by inhibitory feedback from the cerebellar nodulus and uvula [33]. There is also evidence of otolithic 
pathway abnormalities in individuals with VM [34]. It is also suggested that Purkinje cells in the paraflocculus could be inhibited after the occurrence of a migraine episode, which may be an important factor leading to vestibular migraine [35]. Magnetic resonance studies have shown an increased thalamic activation in VM patients during vestibular stimulation compared to healthy controls, as well as grey matter volume abnormalities of nociceptive and multisensory vestibular brain areas [36, 37]. In addition, a PET study during an attack revealed increased metabolism in the temporo-parieto-insular areas and bilateral thalami as well as decreased metabolism in the occipital cortex [38]. Another pathophysiological mechanism in VM may be a visuo-vestibular problem, as patients with VM have a longer duration of post-rotatory nystagmus compared to healthy controls or ordinary migraine patients [39]. Plus, a genetic component in the pathogenesis of VM has been identified $[28,40]$.

\section{Diagnostic criteria}

A VM diagnosis is based on clinical history. The International Headache Society (IHS) and the Barany Society have published a consensus document with diagnostic criteria for vestibular migraine added to the ICHD-3 beta (Appendix, subchapter 'Episodic syndromes that may be associated with migraine') $[4,41]$ (Tab. 1). According to academic panels, VM was defined as an emerging condition needing further research for full validation [4].

The basic diagnostic criteria are presented in Table 1, but some terms need to be broadly described. First of all, vestibular symptoms (as defined by the Bárány Society's Classification of Vestibular Symptoms) include: spontaneous vertigo (internal - a false sensation of self-motion, or external - a false sensation that the visual surrounding is spinning or flowing); positional vertigo; visually-induced vertigo; head motion-induced vertigo; and head motion-induced dizziness with nausea. It is worth noting that to fulfill diagnostic criteria dizziness must be characterised by a sensation of disturbed spatial orientation, thus other forms of dizziness are currently not included in the classification of vestibular migraine [4]. Besides, vestibular symptoms do not include presyncope symptoms, mental confusion, depersonalisation, generalised weakness or fatigue. Moderate vestibular symptoms are defined as those interfering with, but not preventing, daily activities, while severe symptoms are those that stop daily activities. Although vestibular symptoms must last between five minutes and 72 hours, individuals with attacks lasting only for a few seconds may be included if the total period during which short attacks recur is one of at least five minutes [4]. Interestingly, Abouzari et al. compared patients with a diagnosis of VM by ICHD-3 to those who did not fulfill ICHD-3 criteria although they had concurrent vertigo and migraine. They found that both groups were very similar in their characteristics. Thus, adhering strictly to the ICHD-3 criteria, a nearly equivalent
Table 1. Diagnostic criteria for definite vestibular migraine $[4,41]$ Diagnostic criteria for definite vestibular migraine

A. At least five episodes fulfilling criteria $C$ and $D$

B. Current or previous history of migraine with or without aura according to International Classification of Headache Disorders (ICHD-3)

C. Vestibular symptoms of moderate or severe intensity, lasting between 5 minutes and 72 hours

D. One or more migraine features with at least $50 \%$ of the vestibular episodes:

- Headache with at least two of the following characteristics: unilateral location, pulsating quality, moderate or severe pain intensity, aggravation by routine physical activity

- Photophobia and phonophobia

- Visual aura

E. Not better accounted for by another ICHD-3 diagnosis or by another vestibular disorder.

In order to diagnose probable VM, only one of criteria B and D is required to be fulfilled

population that might benefit from migraine treatment would be missed. The authors concluded that the differences between cohorts represented selection bias, not meaningful features, so both groups may represent a spectrum of the same disease [42].

\section{Clinical features}

\section{Vestibular symptoms}

Most patients report internal vertigo (73\%) and triggered vertigo, while external vertigo and positional vertigo are less frequent $[16,18]$. Vertigo is often described as a spinning, rocking, tilting, swaying and falling sensation. According to other study, vestibular symptoms were mainly described as like feeling the ground slipping (40.6\%), swaying (27.7\%), rocking as on a boat (26.7\%), or stepping into an empty space (24.8\%) [15]. Less common vertigo sensations include floating, shimmering, tumbling, bobbing, swimming, sliding, and multi-directional motion [18]. One third of patients experienced unsteadiness [16]. It is worth noting that most patients report more than one vestibular symptom during VM episodes [16]. Almost half of individuals complain of persistent dizziness during the interictal period [16]. The majority of patients continue to have recurrent vertigo in the long-term evolution of VM, with possible slow progression of vestibulo-cochlear dysfunction and the development of mild persistent unsteadiness [43].

\section{Headache and other migraine features}

Although the core of the disease is migraine, and according to diagnostic criteria migraine features must be present for at least half of the time along with vestibular symptoms, in some VM patients, headache and vestibular symptoms never occur together.

In cases without headache, other associated features like age at onset and duration of episodes indicate that vertigo is a migraine equivalent. Headaches accompany VM episodes in almost $50 \%$ of patients; some report head fullness or head 
pressure without headache [16]. Interestingly, headaches during VM episodes are usually less severe compared to a typical migraine-related headache, thus patients are more bothered by the vertigo than by the headache [23]. Balci et al. [44] confirmed that headache severity was higher in migraineurs than in vestibular migraineurs. During VM episodes, other migraine features are also observed, often without a headache, including nausea, photophobia, phonophobia, osmophobia, allodynia and vomiting $[5,18,42]$. If a patient complains of nausea and vomiting during their typical migraine headaches, they tend to experience similar symptoms during VM attacks more frequently [8]. The higher prevalence of migraine with aura (MA) seen in VM patients may reflect an association of MA with vascular disease and posterior circulation [22]. We would like to underline that patients with vertigo should be directly asked about migraine symptoms during their episodes, as they often do not volunteer them [5].

\section{Other symptoms}

Some patients report tinnitus, fullness of the ear, palpitations, and even mild and transient hearing loss, during their episodes [18]. Several authors have described an accompanying Alice in Wonderland Syndrome - a rare and fascinating sensory perception disorder, with the symptoms of visual distortions, extrapersonal misperceptions, or somesthetic distortions $[16,45]$. Some studies show that VM patients are significantly more anxious and agoraphobic, have depression symptoms and insomnia, have higher sensitivity to separation, and are prone to seek medical reassurance $[16,46]$.

\section{Duration of vestibular attacks}

The duration of episodes varies from a few seconds up to several days $[8,18]$. According to Vuralli et al. [15], vestibular symptoms were described as short attacks lasting for seconds by $60.4 \%$ of VM patients, but the total time with repeated short episodes lasted between hours and days. Notably, patients with short-lasting attacks tend to have several such attacks every day $[15,19]$.

\section{Triggers and aggravating factors}

The top VM triggers are stress, sleep deprivation, bright lights, missing meals and weather changes; these do not differ from typical migraine triggers [16, 47]. Some attacks may be started by daily head and body movements, menstruation and sensory stimuli $[5,15]$. Visually induced vestibular symptoms have been described by $71.3 \%$ of patients, and positional motion-induced vestibular symptoms by $82.2 \%$ of patients [15].

\section{Neuro-otological examination}

A neurological as well as an otological examination is usually normal between episodes, although mild central deficits can exist, mostly in patients with a long history of VM $[14,43,48]$. During VM episodes, most patients have a central spontaneous or positional nystagmus $[48,49]$. The nystagmus has no latency period, is usually persistent and non-fatigable, and is markedly reduced by visual fixation [50,51]. In addition, imbalance is a regular finding during acute attacks, including a positive Romberg test and gait ataxia [17, 49].

\section{Differential diagnosis}

VM must be differentiated particularly from Meniere's Disease (MD) and benign paroxysmal peripheral vertigo (BPPV). Regardless of the many differences between these disorders, one should remember that, at very early stages, most symptoms can be quite similar. Meniere's Disease is a disorder with attacks of coexisting vertigo, tinnitus, aural fullness, and hearing loss [32]. MD is known to be associated with migraine, as the lifetime prevalence of migraine is higher in the MD group compared to controls, and almost half of MD patients may experience headache, photophobia or aura during Meniere's attacks [32, 52]. Radtke et al. [52] reported that the lifetime prevalence of migraine was higher in the MD group (56\%) than controls $(25 \%, \mathrm{p}<0.001)$; they concluded that migraine and MD might share a similar pathophysiology.

In a very large group of 911 MD patients, Pyykkö et al. [53] found migraine in $20.9 \%$ of subjects while headache was reported by $42.9 \%$. Notably, migraine patients complained of more severe $\mathrm{MD}$ symptoms than non-migraineur patients with MD. Shin et al. [54] discovered an incidence of VM in 35\% of MD patients, mostly with a probable MD diagnosis. One study revealed that MD patients with VM have better hearing and more frequent vertigo episodes than individuals without VM [55]. Interestingly, some patients may experience the clinical features of both VM and MD during their attack. This has been described in the literature as ' $\mathrm{VM} / \mathrm{MD}$ overlapping syndrome' [56].

Nevertheless, many differences between these two disorders exist, as individuals with MD suffer mostly from accompanying auditory symptoms, anxiety, and palpitations, while migraine sufferers experience typical headache, photo-I phonophobia or aura $[57,58]$. Endolymphatic hydrops is the primary pathological finding in MD patients, although Gurkov et al. [59] using MRI found endolymphatic hydrops in $20 \%$ of patients with VM and auditory symptoms. Also, other vestibular tests do not completely segregate individuals with $\mathrm{MD}$ from those with VM $[60,61]$.

BPPV is another common cause of vertigo and should be considered in the differential diagnosis of VM patients. Notably, migraine is more common in patients with BPPV compared to controls, and having migraine is linked with an increased risk of developing BPPV [11, 14]. For differentiation, positional testing which stimulates vertiginous attacks and elicits nystagmus may be helpful, including the Dix-Hallpike manoeuvre or the supine roll test for the horizontal canal variant [62].

Other disorders that rarely may mimic VM, and should be taken into consideration during the differentiation process, include: vertebrobasilar TIA, vascular compression of 
the eighth nerve, autoimmune inner ear disease, insufficient compensation of unilateral vestibular loss, schwannoma of the eighth nerve, persistent postural-perceptual dizziness, and anxiety disorder $[16,26,62]$.

\section{Laboratory findings}

Although no pathognomonic laboratory test that can verify a VM diagnosis exists, investigations should be done to rule out any other diagnosis. Many authors report minor signs of peripheral and central vestibular dysfunction, including pathological results in caloric and video head impulse testing (vHIT) as well as in tonal audiometry, cervical and ocular vestibular evoked myogenic potentials (VEMP), posturography or magnetic resonance imaging (MRI), but the findings are ambiguous [61, 63-71].

It is worth emphasising that abnormal vestibular testing results are also found in patients with migraine without a history of vestibular symptoms [72]. In most patients, tonal audiometry is normal [73]. The most consistent laboratory finding in VM is a unilaterally reduced caloric response as well as bilateral caloric hyporesponsiveness, but only in a small percentage of patients.

Notably, individuals with VM are significantly more likely to have an emetic response to caloric stimulation than other patients [74]. Almost half of VM patients have saccadic movements in vHIT [10]. Some studies have revealed that cervical VEMP can be used as a diagnostic test to differentiate MD from VM, because VEMP responses are symmetrically reduced on both sides in VM patients [75]. One study has demonstrated an absence of unilateral or bilateral VEMP response at $90 \mathrm{~dB}$ normal hearing level in almost half of VM patients, which was significantly higher than in healthy controls [65], while another showed reduced VEMP amplitudes compared to controls, suggesting that not only peripheral but also central vestibular structures are affected in VM patients [63].

Moreover, patients with VM have shown compromised body balance in static posturography tests, and higher visual dependency and low stability of the postural control system when maintaining quiet standing $[67,76]$. Brain MRI is unremarkable in VM sufferers, although some studies found that grey matter volume of some brain regions of patients with $\mathrm{VM}$ is significantly larger than in patients with migraine or healthy controls, mostly areas linked with assessment, integration and pain expectations as well as those associated with mood and anxiety $[16,77]$. Interestingly, other authors have found grey matter volume reduction in the temporal gyrus, mid-cingulate, dorsolateral prefrontal area, insula, parietal and occipital cortex [78].

\section{Vestibular migraine treatment}

Due to the lack of well-conducted randomised clinical trials, VM treatment recommendations are mostly based on the guidelines for migraine therapy or observational studies, retrospective cohorts and open-label trials, anecdotal experience, and expert opinion [79-81]. We should remember that inconsistent definitions of VM based on old diagnostic criteria were used in many of these studies, thus examined cohorts were quite heterogeneous. Also, because of the absence of control groups in most of the studies it is difficult to determine if the improvement is spontaneous or due to treatment. Generally, migraine prophylactic treatments were effective in $77 \%$ of VM patients [19]. Surprisingly, even patients with headache and dizziness who did not meet VM diagnostic criteria benefit from prophylactic treatment of VM [82]. Regardless of the wide range of drugs that can be prescribed for the prophylactic treatment of VM, their effectiveness seems to be similar. Domínguez-Durán et al. [83], in a prospective study, reported that after five weeks of treatment, topiramate, amitryptyline, propranolol, flunarisine and acetazolamid were all equally effective in reducing vestibular symptoms, headache and the number of crises. A recent systematic review and meta-analysis revealed that although all treatment options showed improvements in outcome parameters, due to heterogeneity and the lack of standardised reporting on outcomes, no preferred treatment modality could be determined [84].

All studies regarding VM acute or prophylactic treatment (based on the modern VM definition) are summarised in Table 2. Several older studies regarding treatment of so called migraneus vertigo, with inconsistent groups, also exist [85]. They showed the effectiveness of acetazolamide, topiramate, lamotrygine, pizotifen, flunarisine or amitryptyline in reducing vertigo or headache [85]. Apart from pharmacological treatment, other treatments include lifestyle modification, trigger avoidance, and vestibular rehabilitation, although results are inconsistent $[86,87]$. Interestingly, recent trials of specific migraine neuromodulation devices (trigeminal external nerve stimulation and noninvasive vagus nerve stimulation) have reported improvements in vertigo and headache severity without any side effect in the majority of VM patients $[88,89]$. Because in recent times monoclonal anti-CGRP antibodies have been approved for migraine prevention, the question arises as to whether they might be effective in VM sufferers. Theoretically, they could play a role in vestibular physiology, because CGRP is also detected in human cochlear and vestibular end organs, and possibly plays a role in motion sickness $[90,91]$. But as yet there is too little data on this topic.

\section{Clinical implications}

Vestibular migraine is a common cause of vertigo, and may affect $1-3 \%$ of the general population. It is a disabling disease of recurrent attacks of vestibular symptoms accompanied by migraine features and it occurs in patients with a current or previous history of migraine. The episodes can last for minutes, hours or even days, and may occur without a concurrent headache, which can increase misdiagnosis. VM often begins 
(1/

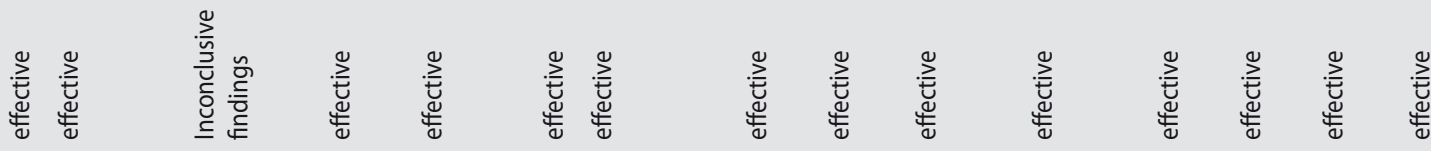

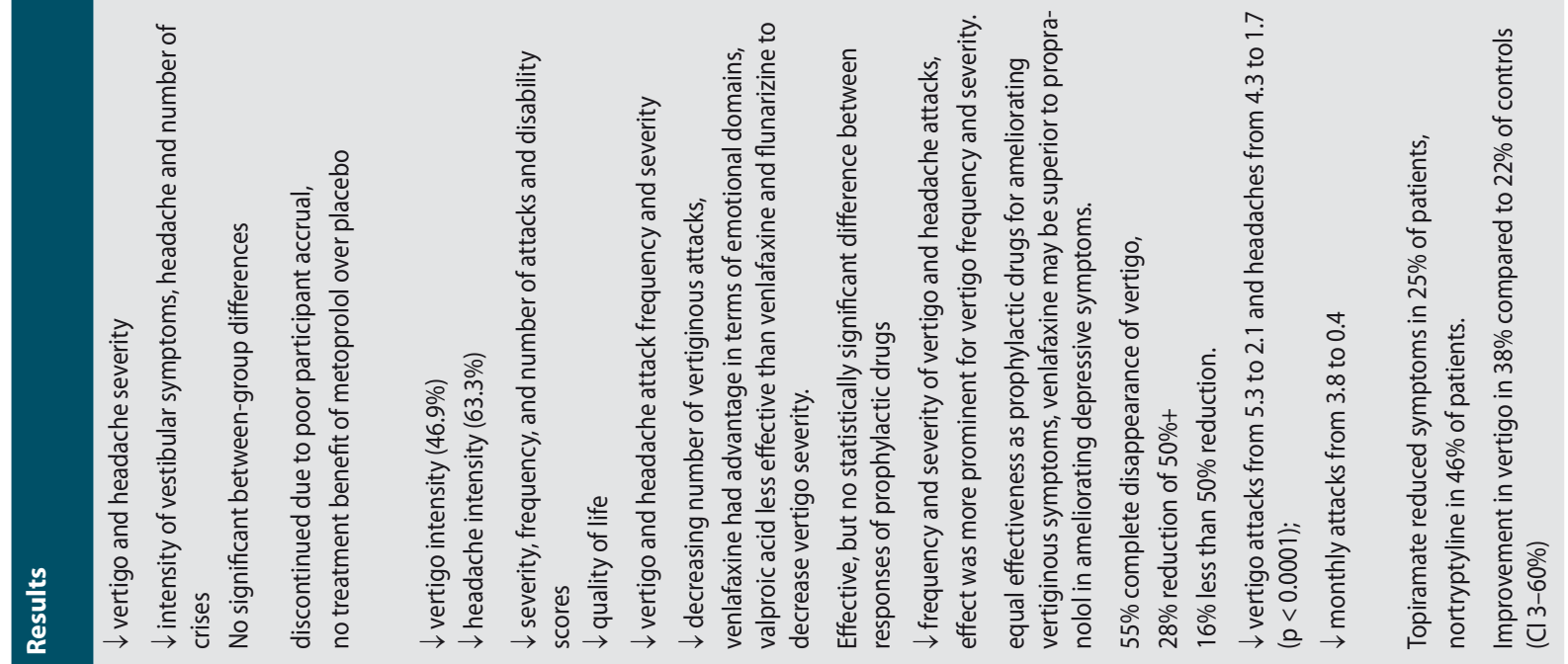

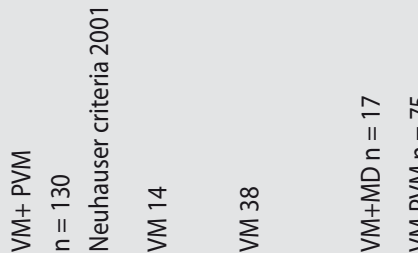

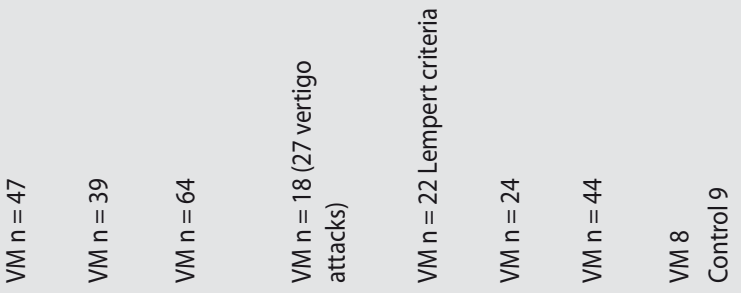

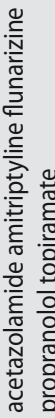

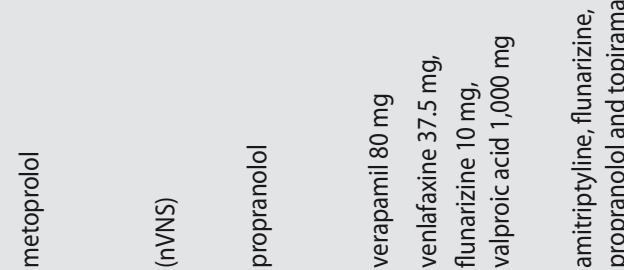

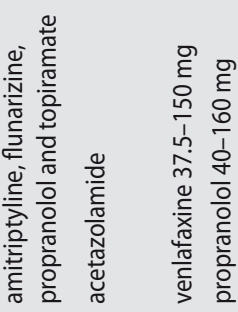

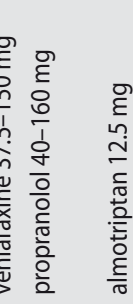

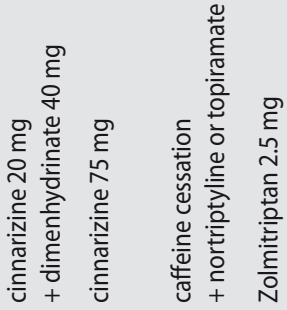

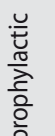

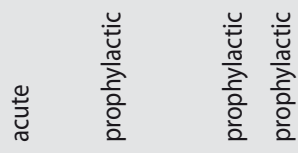

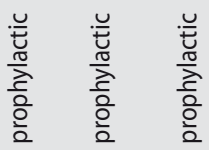

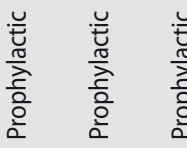

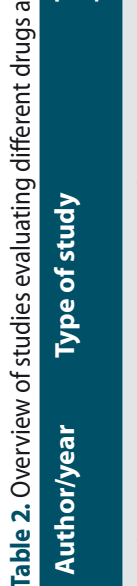

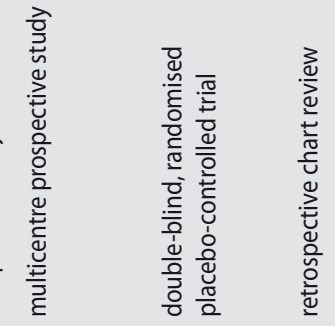

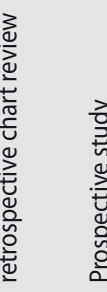

产

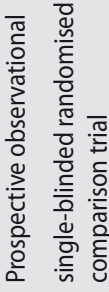

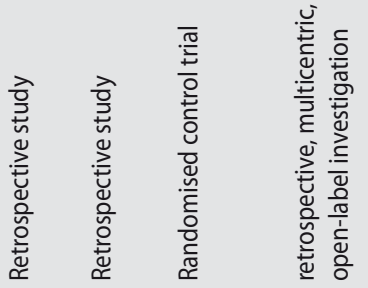

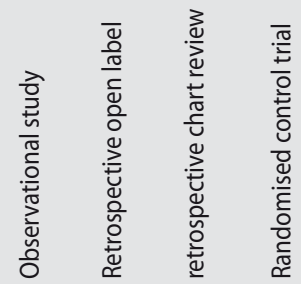

要

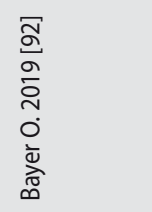

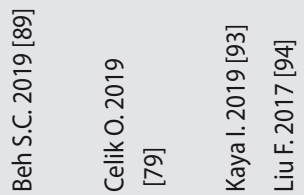

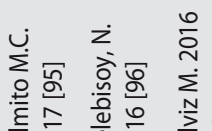

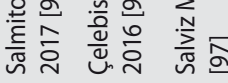


several years after typical migraine and there may be a considerable delay between onset of headache and vertigo. The diagnosis is based on the patient's clinical history.

According to the recent consensus reached by the International Headache Society and the Barany Society, in order to fulfill VM diagnostic criteria, attacks of vestibular symptoms connected with at least one migraine feature must be observed. Diagnosing vestibular migraine is a challenge, as there is lack of established confirmatory tests or biomarkers. The mechanism of vestibular migraine remains unclear and is still under investigation, but there is apparent interaction between the trigeminal and vestibular systems. Due to the lack of specific trials, treatment recommendations are based on migraine guidelines and several drugs seem to be effective, although there have been few randomised controlled trials.

Regardless of the published strict and detailed diagnostic criteria, this condition remains little known, and as a result it is underdiagnosed and undertreated.

Efforts should be made to educate medical communities and patients about this frequent disease and to encourage neurologists and ENT specialists to cooperate. It is important that every patient with vertigo of unknown origin should be directly asked about a past or present history of migraine, and about any migraine symptoms experienced during their vertigo episodes.

In cases with coexisting migraine and vertigo, a VM diagnosis should always be considered.

\section{Future directions}

There is a need for future studies in order to fully validate the diagnostic criteria and better characterise this disorder. Moreover, well conducted randomised trials must be performed to establish clear treatment recommendations. Finally, efforts should be made to elucidate and understand the pathophysiology of VM, from both the clinical and the scientific perspectives, so as to improve management of this surprisingly common disorder.

\section{Conflicts of interest: None}

Ethical approval: Not necessary for the preparation of this article

\section{References}

1. Neuhauser HK, Radtke A, von Brevern M, et al. Migrainous vertigo: prevalence and impact on quality of life. Neurology. 2006; 67(6): 1028-1033, doi: 10.1212/01.wnl.0000237539.09942.06, indexed in Pubmed: 17000973.

2. Vuković $\mathrm{V}$, Plavec $\mathrm{D}$, Galinović I, et al. Prevalence of vertigo, dizziness, and migrainous vertigo in patients with migraine. Headache. 2007; 47(10): 1427-1435, doi: 10.1111/j.1526-4610.2007.00939.x, indexed in Pubmed: 18052952.
3. Calhoun AH, Ford S, Pruitt AP, et al. The point prevalence of dizziness or vertigo in migraine--and factors that influence presentation. Headache. 2011; 51(9): 1388-1392, doi: 10.1111/j.1526-4610.2011.01970.x, indexed in Pubmed: 21797862.

4. Headache Classification Committee of the International Headache Society (IHS) The International Classification of Headache Disorders, 3rd edition. Cephalalgia. 2018; 38(1): 1-211, doi: 10.1177/0333102417738202.

5. Lempert T, Brevern Mv. Vestibular Migraine. Neurol Clin. 2019; 37(4): 695-706, doi: 10.1016/j.ncl.2019.06.003.

6. Geser R, Straumann D. Referral and final diagnoses of patients assessed in an academic vertigo center. Front Neurol. 2012; 3: 169, doi: 10.3389/fneur.2012.00169, indexed in Pubmed: 23226141.

7. Formeister EJ, Rizk HG, Kohn MA, et al. The Epidemiology of Vestibular Migraine: A Population-based Survey Study. Otol Neurotol. 2018; 39(8): 1037-1044, doi: 10.1097/MA0.0000000000001900, indexed in Pubmed: 30020261.

8. Teggi R, Colombo B, Albera R, et al. Clinical Features, Familial History, and Migraine Precursors in Patients With Definite Vestibular Migraine: The VM-Phenotypes Projects. Headache. 2018; 58(4): 534-544, doi: 10.1111/head.13240, indexed in Pubmed: 29205326.

9. Cho SJ, Kim BK, Kim BS, et al. Vestibular migraine in multicenter neurology clinics according to the appendix criteria in the third beta edition of the International Classification of Headache Disorders. Cephalalgia. 2016; 36(5): 454-462, doi: 10.1177/0333102415597890, indexed in Pubmed: 26224714.

10. Yollu U, Uluduz DU, Yilmaz M, et al. Vestibular migraine screening in a migraine-diagnosed patient population, and assessment of vestibulocochlear function. Clin Otolaryngol. 2017; 42(2): 225-233, doi: 10.1111/coa.12699, indexed in Pubmed: 27385658.

11. Cha YH, Lee H, Santell LS, et al. Association of benign recurrent vertigo and migraine in 208 patients. Cephalalgia. 2009; 29(5): 550-555, doi: 10.1111/j.1468-2982.2008.01770.x, indexed in Pubmed: 19170697.

12. Martinez E, Ruiz-Pinero $M$, de Lera $M$, et al. [Clinical characteristics of vestibular migraine: considerations in a series of 41 patients]. Rev Neurol. 2017; 64(1): 1-6, indexed in Pubmed: 28000906.

13. Neuhauser $\mathrm{H}$, Leopold $M$, von Brevern $M$, et al. The interrelations of migraine, vertigo, and migrainous vertigo. Neurology. 2001; 56(4): 436441, doi: 10.1212/wnl.56.4.436, indexed in Pubmed: 11222783.

14. Dieterich M, Obermann M, Celebisoy N. Vestibular migraine: the most frequent entity of episodic vertigo. J Neurol. 2016; 263 Suppl 1: S82-S89, doi: 10.1007/s00415-015-7905-2, indexed in Pubmed: 27083888.

15. Vuralli D, Yildirim F, Akcali DT, et al. Visual and Postural Motion-Evoked Dizziness Symptoms Are Predominant in Vestibular Migraine Patients. Pain Med. 2018; 19(1): 178-183, doi: 10.1093/pm/pnx182, indexed in Pubmed: 29017000.

16. Beh SC, Masrour S, Smith SV, et al. The Spectrum of Vestibular Migraine: Clinical Features, Triggers, and Examination Findings. Headache. 2019; 59(5): 727-740, doi: 10.1111/head.13484, indexed in Pubmed: 30737783.

17. Power L, Shute W, McOwan B, et al. Clinical characteristics and treatment choice in vestibular migraine. J Clin Neurosci. 2018; 52: 50-53, doi: 10.1016/i.jocn.2018.02.020, indexed in Pubmed: 29550250.

18. Teggi R, Colombo B, Albera R, et al. Clinical Features of Headache in Patients With Diagnosis of Definite Vestibular Migraine: The 
VM-Phenotypes Projects. Front Neurol. 2018; 9, doi: 10.3389/ fneur.2018.00395.

19. Zhang Y, Kong Q, Chen J, et al. International Classification of Headache Disorders 3rd edition beta-based field testing of vestibular migraine in China: Demographic, clinical characteristics, audiometric findings and diagnosis statues. Cephalalgia. 2016; 36(3): 240-248, doi: 10.1177/0333102415587704, indexed in Pubmed: 25986149.

20. Lempert T, Neuhauser H. Epidemiology of vertigo, migraine and vestibular migraine. J Neurol. 2009; 256(3): 333-338, doi: $10.1007 /$ s00415-009-0149-2.

21. Thakar A, Anjaneyulu C, Deka RC. Vertigo syndromes and mechanisms in migraine. J Laryngol Otol. 2001; 115(10): 782-787, doi: 10.1258/0022215011909251, indexed in Pubmed: 11667987.

22. Baloh RW. Vestibular Migraine I: Mechanisms, Diagnosis, and Clinical Features. Semin Neurol. 2020; 40(1): 76-82, doi: 10.1055/s-00393402735, indexed in Pubmed: 31935766.

23. Colombo B, Teggi R. NIVE Project. Vestibular migraine: who is the patient? Neurol Sci. 2017; 38(Suppl 1): 107-110, doi: $10.1007 /$ s10072-017-2882-0, indexed in Pubmed: 28527080.

24. Chang TP, Hsu YC. Vestibular migraine has higher correlation with carsickness than non-vestibular migraine and Meniere's disease. Acta Neurol Taiwan. 2014; 23(1): 4-10, indexed in Pubmed: 24833209.

25. Beh SC. Vestibular Migraine: How to Sort it Out and What to Do About it. J Neuroophthalmol. 2019; 39(2): 208-219, doi: 10.1097/ WN0.0000000000000791, indexed in Pubmed: 31094996.

26. Popkirov S, Staab JP, Stone J. Persistent postural-perceptual dizziness (PPPD): a common, characteristic and treatable cause of chronic dizziness. Pract Neurol. 2018; 18(1): 5-13, doi: 10.1136/practneurol-2017-001809, indexed in Pubmed: 29208729.

27. Millen SJ, Schnurr CM, Schnurr BB. Vestibular migraine: perspectives of otology versus neurology. Otol Neurotol. 2011; 32(2): 330-337, doi: 10.1097/MAO.0b013e3182040b21, indexed in Pubmed: 21178806.

28. Furman JM, Marcus DA, Balaban CD. Vestibular migraine: clinical aspects and pathophysiology. Lancet Neurol. 2013; 12(7): 706-715, doi: 10.1016/S1474-4422(13)70107-8, indexed in Pubmed: 23769597.

29. Espinosa-Sanchez JM, Lopez-Escamez JA. New insights into pathophysiology of vestibular migraine. Front Neurol. 2015; 6: 12, doi: 10.3389/fneur.2015.00012, indexed in Pubmed: 25705201.

30. Kreczmański P, Wolak T, Lewandowska M, et al. Altered functional brain imaging in migraine patients: BOLD preliminary study in migraine with and without aura. Neurol Neurochir Pol. 2019; 53(4): 304-310, doi: 10.5603/PJNNS.a2019.0035, indexed in Pubmed: 31441496.

31. Balaban CD, Black RD, Silberstein SD. Vestibular Neuroscience for the Headache Specialist. Headache. 2019; 59(7): 1109-1127, doi: 10.1111/head.13550, indexed in Pubmed: 31077365.

32. Liu YF, Xu H. The Intimate Relationship between Vestibular Migraine and Meniere Disease: A Review of Pathogenesis and Presentation. Behav Neurol. 2016; 2016: 3182735, doi: 10.1155/2016/3182735, indexed in Pubmed: 27651559.

33. King S, Priesol AJ, Davidi SE, et al. Self-motion perception is sensitized in vestibular migraine: pathophysiologic and clinical implications. Sci Rep. 2019; 9(1): 14323, doi: 10.1038/s41598-019-50803-y, indexed in Pubmed: $\underline{31586151}$.

34. Ashish G, Augustine AM, Tyagi AK, et al. Subjective Visual Vertical and Horizontal in Vestibular Migraine. J Int Adv Otol. 2017; 13(2): 254-258, doi: 10.5152/iao.2017.4056, indexed in Pubmed: 28816696.
35. Li P, Gu H, Xu J, et al. Purkinje cells of vestibulocerebellum play an important role in acute vestibular migraine. J Integr Neurosci. 2019; 18(4): 409-414, doi: 10.31083/j.jin.2019.04.1168, indexed in Pubmed: 31912699.

36. Russo A, Marcelli V, Esposito F, et al. Abnormal thalamic function in patients with vestibular migraine. Neurology. 2014; 82(23): 2120-2126, doi: 10.1212/WNL.0000000000000496, indexed in Pubmed: 24814847.

37. Messina R, Rocca MA, Colombo B, et al. Structural brain abnormalities in patients with vestibular migraine. J Neurol. 2017; 264(2): 295-303, doi: 10.1007/s00415-016-8349-z, indexed in Pubmed: 27888414.

38. Shin JH, Kim YuK, Kim HJ, et al. Altered brain metabolism in vestibular migraine: comparison of interictal and ictal findings. Cephalalgia. 2014; 34(1): 58-67, doi: 10.1177/0333102413498940, indexed in Pubmed: 23918837.

39. Jeong $\mathrm{SH}$, Oh SY, Kim HJ, et al. Vestibular dysfunction in migraine: effects of associated vertigo and motion sickness. J Neurol. 2010; 257(6): 905-912, doi: 10.1007/s00415-009-5435-5, indexed in Pubmed: 20041331.

40. Wu X, Qiu F, Wang Z, et al. Correlation of 5-HTR6 gene polymorphism with vestibular migraine. J Clin Lab Anal. 2020; 34(2): e23042, doi: 10.1002/jcla.23042, indexed in Pubmed: 31587366.

41. Lempert T, Olesen J, Furman J, et al. Vestibular migraine: diagnostic criteria. J Vestib Res. 2012; 22(4): 167-172, doi: 10.3233/VES2012-0453, indexed in Pubmed: 23142830.

42. Abouzari M, Goshtasbi K, Moshtaghi O, et al. Association Between Vestibular Migraine and Migraine Headache: Yet to Explore. Otol Neurotol. 2020; 41(3): 392-396, doi: 10.1097/MAO.0000000000002528, indexed in Pubmed: 31821258.

43. Radtke A, von Brevern M, Neuhauser $\mathrm{H}$, et al. Vestibular migraine: long-term follow-up of clinical symptoms and vestibulo-cochlear findings. Neurology. 2012; 79(15): 1607-1614, doi: $10.1212 /$ WNL.0b013e31826e264f, indexed in Pubmed: 23019266.

44. Balci B, Şenyuva N, Akdal G. Definition of Balance and Cognition Related to Disability Levels in Vestibular Migraine Patients. Noro Psikiyatr Ars. 2018; 55(1): 9-14, doi: 10.29399/npa.12617, indexed in Pubmed: $\underline{30042635}$.

45. Beh SC, Masrour S, Smith SV, et al. Clinical characteristics of Alice in Wonderland syndrome in a cohort with vestibular migraine. Neurol Clin Pract. 2018; 8(5): 389-396, doi: 10.1212/ CPJ.0000000000000518, indexed in Pubmed: 30564492.

46. Kutay 0̈, Akdal G, Keskinoğlu P, et al. Vestibular migraine patients are more anxious than migraine patients without vestibular symptoms. J Neurol. 2017; 264(Suppl 1): 37-41, doi: 10.1007/s00415-0178439-6, indexed in Pubmed: 28280987.

47. Chądzyński P, Kacprzak A, Domitrz W, et al. Migraine headache facilitators in a population of Polish women and their association with migraine occurrence - preliminary results. Neurol Neurochir Pol. 2019; 53(5): 377-383, doi: 10.5603/PJNNS.a2019.0044, indexed in Pubmed: 31592536.

48. Neugebauer H, Adrion C, Glaser M, et al. Long-term changes of central ocular motor signs in patients with vestibular migraine. Eur Neurol. 2013; 69(2): 102-107, doi: 10.1159/000343814, indexed in Pubmed: 23182929.

49. von Brevern $M$, Zeise $D$, Neuhauser $H$, et al. Acute migrainous vertigo: clinical and oculographic findings. Brain. 2005; 128(Pt 2): 365-374, doi: 10.1093/brain/awh351, indexed in Pubmed: 15601663. 
50. El-Badry MM, Samy H, Kabel AM, et al. Clinical criteria of positional vertical nystagmus in vestibular migraine. Acta Otolaryngol. 2017; 137(7): 720-722, doi: 10.1080/00016489.2017.1318220, indexed in Pubmed: 28446038.

51. Dieterich M, Brandt T. Episodic vertigo related to migraine (90 cases): vestibular migraine? J Neurol. 1999; 246(10): 883-892, doi: 10.1007/s004150050478, indexed in Pubmed: 10552234.

52. Radtke A, Lempert T, Gresty MA, et al. Migraine and Ménière's disease: is there a link? Neurology. 2002; 59(11): 1700-1704, doi: 10.1212/01.wnl.0000036903.22461.39, indexed in Pubmed: 12473755.

53. Pyykkö I, Pyykkö N, Manchaiah V, et al. Association between Ménière's disease and vestibular migraine. Auris Nasus Larynx. 2019; 46(5): 724-733, doi: 10.1016/i.anl.2019.02.002, indexed in Pubmed: 31054848.

54. Shin $\mathrm{CHo}$, Kim Y, Yoo MH, et al. Management of Ménière's Disease: How Does the Coexistence of Vestibular Migraine Affect Outcomes? Otol Neurotol. 2019; 40(5): 666-673, doi: 10.1097/ MAO.0000000000002176, indexed in Pubmed: 31083096.

55. Wang Y, Diao T, Zhao Y, et al. The clinical characteristics and audiogram in 103 Meniere's disease patients with and without vestibular migraine. Clin Otolaryngol. 2018; 43(1): 343-347, doi: 10.1111/ coa.12946, indexed in Pubmed: 28742261.

56. Murofushi T, Tsubota M, Kitao K, et al. Simultaneous Presentation of Definite Vestibular Migraine and Definite Ménière's Disease: Overlapping Syndrome of Two Diseases. Front Neurol. 2018; 9: 749, doi: 10.3389/fneur.2018.00749, indexed in Pubmed: 30250448.

57. Lopez-Escamez JA, Dlugaiczyk J, Jacobs J, et al. Accompanying Symptoms Overlap during Attacks in Menière's Disease and Vestibular Migraine. Front Neurol. 2014; 5: 265, doi: 10.3389/ fneur.2014.00265, indexed in Pubmed: 25566172.

58. Tabet P, Saliba I. Meniere's Disease and Vestibular Migraine: Updates and Review of the Literature. J Clin Med Res. 2017; 9(9): 733-744, doi: 10.14740/jocmr3126w, indexed in Pubmed: 28811849.

59. Gürkov R, Kantner C, Strupp M, et al. Endolymphatic hydrops in patients with vestibular migraine and auditory symptoms. Eur Arch Otorhinolaryngol. 2014; 271(10): 2661-2667, doi: 10.1007/s00405013-2751-2, indexed in Pubmed: 24121780.

60. Zuniga MG, Janky KL, Schubert MC, et al. Can vestibular-evoked myogenic potentials help differentiate Ménière disease from vestibular migraine? Otolaryngol Head Neck Surg. 2012; 146(5): 788-796, doi: 10.1177/0194599811434073, indexed in Pubmed: 22267492.

61. Blödow A, Heinze M, Bloching MB, et al. Caloric stimulation and video-head impulse testing in Ménière's disease and vestibular migraine. Acta Otolaryngol. 2014; 134(12): 1239-1244, doi: 10.3109/00016489.2014.939300, indexed in Pubmed: 25399882.

62. Cohen JM, Escasena CA. Headache and Dizziness: How to Differentiate Vestibular Migraine from Other Conditions. Curr Pain Headache Rep. 2015; 19(7): 31, doi: 10.1007/s11916-015-0502-3, indexed in Pubmed: 26049771.

63. Baier B, Stieber N, Dieterich M. Vestibular-evoked myogenic potentials in vestibular migraine. J Neurol. 2009; 256(9): 1447-1454, doi: 10.1007/s00415-009-5132-4, indexed in Pubmed: 19377861.

64. Bednarczuk NF, Bonsu A, Ortega MC, et al. Abnormal visuo-vestibular interactions in vestibular migraine: a cross sectional study. Brain. 2019; 142(3): 606-616, doi: 10.1093/brain/awy355, indexed in Pubmed: 30759189.
65. Boldingh MI, Ljøstad U, Mygland A, et al. Vestibular sensitivity in vestibular migraine: VEMPs and motion sickness susceptibility. Cephalalgia. 2011; 31(11): 1211-1219, doi: 10.1177/0333102411409074, indexed in Pubmed: 21768183.

66. Cesaroni S, Silva AM, Ganança MM, et al. Postural control at posturography with virtual reality in the intercritical period of vestibular migraine. Braz J Otorhinolaryngol. 2019 [Epub ahead of print], doi: 10.1016/j.bjorl.2019.06.015, indexed in Pubmed: 31439532.

67. Gorski LP, Silva AM, Cusin FS, et al. Body balance at static posturography in vestibular migraine. Braz J Otorhinolaryngol. 2019; 85(2): 183-192, doi: 10.1016/j.bjorl.2017.12.001, indexed in Pubmed: 29370980.

68. Inoue A, Egami N, Fujimoto C, et al. Vestibular Evoked Myogenic Potentials in Vestibular Migraine: Do They Help Differentiating From Menière's Disease? Ann Otol Rhinol Laryngol. 2016; 125(11): 931 937, doi: 10.1177/0003489416665192, indexed in Pubmed: 27557910.

69. Kang WS, Lee SH, Yang CJ, et al. Vestibular Function Tests for Vestibular Migraine: Clinical Implication of Video Head Impulse and Caloric Tests. Front Neurol. 2016; 7: 166, doi: 10.3389/fneur.2016.00166, indexed in Pubmed: 27746761.

70. Makowiec KF, Piker EG, Jacobson GP, et al. Ocular and Cervical Vestibular Evoked Myogenic Potentials in Patients With Vestibular Migraine. Otol Neurotol. 2018; 39(7): e561-e567, doi: 10.1097/ MAO.0000000000001880, indexed in Pubmed: 29912833.

71. Sharon JD, Hullar TE. Motion sensitivity and caloric responsiveness in vestibular migraine and Meniere's disease. Laryngoscope. 2014; 124(4): 969-973, doi: 10.1002/lary.24285, indexed in Pubmed: 23818082.

72. Boldingh MI, Ljøstad U, Mygland A, et al. Comparison of interictal vestibular function in vestibular migraine vs migraine without vertigo. Headache. 2013; 53(7): 1123-1133, doi: 10.1111/head.12129, indexed in Pubmed: 23676003.

73. Morganti LO, Salmito MC, Duarte JA, et al. Vestibular migraine: clinical and epidemiological aspects. Braz J Otorhinolaryngol. 2016; 82(4): 397-402, doi: 10.1016/j.bjorl.2015.06.003, indexed in Pubmed: 26614042.

74. Vitkovic J, Paine M, Rance G. Neuro-otological findings in patients with migraine- and nonmigraine-related dizziness. Audiol Neurootol. 2008; 13(2): 113-122, doi: 10.1159/000111783, indexed in Pubmed: 18057875.

75. Salviz M, Yuce T, Acar H, et al. Diagnostic value of vestibular-evoked myogenic potentials in Ménière's disease and vestibular migraine. $J$ Vestib Res. 2016; 25(5-6): 261-266, doi: 10.3233/VES-160567. indexed in Pubmed: 26890427.

76. Lim YH, Kim JS, Lee HW, et al. Postural Instability Induced by Visual Motion Stimuli in Patients With Vestibular Migraine. Front Neurol. 2018; 9: 433, doi: 10.3389/fneur.2018.00433, indexed in Pubmed: 29930534.

77. Wang $\mathrm{S}$, Wang $\mathrm{H}$, Zhao D, et al. Grey matter changes in patients with vestibular migraine. Clin Radiol. 2019; 74(11): 898.e1-898.e5, doi: 10.1016/i.crad.2019.07.015, indexed in Pubmed: 31451181.

78. Obermann M, Wurthmann S, Steinberg BS, et al. Central vestibular system modulation in vestibular migraine. Cephalalgia. 2014; 34(13): 1053-1061, doi: $10.1177 / 0333102414527650$, indexed in Pubmed: 24662323.

79. Çelik O, Tanyeri Toker G, Eskiizmir G, et al. The Effectiveness of Medical Prophylactic Treatment on Vestibular Migraine and Its Effect on 
the Quality Of Life. J Int Adv Otol. 2019 [Epub ahead of print], doi: 10.5152/iao.2019.6522, indexed in Pubmed: 31347507.

80. Domínguez-Durán E, Baños-López P, Martín-Castillo E, et al. Interrater agreement in the choice of prophylactic treatment for vestibular migraine and role of an assisted algorithm for this choice. Acta Otorrinolaringol Esp. 2019 [Epub ahead of print], doi: 10.1016/j. otorri.2019.04.002, indexed in Pubmed: 31420102.

81. Lauritsen CG, Marmura MJ. Current Treatment Options: Vestibular Migraine. Curr Treat Options Neurol. 2017; 19(11): 38, doi: 10.1007/ s11940-017-0476-z, indexed in Pubmed: 28965306.

82. Salmito MC, Morganti LO, Nakao BH, et al. Vestibular migraine: comparative analysis between diagnostic criteria. Braz J Otorhinolaryngol. 2015; 81(5): 485-490, doi: 10.1016/j.bjorl.2015.07.007, indexed in Pubmed: 26277830.

83. Domínguez-Durán E, Montilla-lbáñez MA, Álvarez-Morujo de Sande $M G$, et al. Analysis of the effectiveness of the prophylaxis of vestibular migraine depending on the diagnostic category and the prescribed drug. Eur Arch Otorhinolaryngol. 2020; 277(4): 1013-1021, doi: 10.1007/s00405-020-05802-5, indexed in Pubmed: 32008074.

84. Byun YJ, Levy DA, Nguyen SA, et al. Treatment of Vestibular Migraine: A Systematic Review and Meta-analysis. Laryngoscope. 2020 [Epub ahead of print], doi: 10.1002/lary.28546, indexed in Pubmed: 32083732.

85. Fotuhi M, Glaun B, Quan SY, et al. Vestibular migraine: a critical review of treatment trials. J Neurol. 2009; 256(5): 711-716, doi: 10.1007/ s00415-009-5050-5, indexed in Pubmed: 19252785.

86. Alghadir AH, Anwer S. Effects of Vestibular Rehabilitation in the Management of a Vestibular Migraine: A Review. Front Neurol. 2018; 9: 440, doi: 10.3389/fneur.2018.00440, indexed in Pubmed: 29946294.

87. Sugaya N, Arai M, Goto F. Is the Headache in Patients with Vestibular Migraine Attenuated by Vestibular Rehabilitation? Front Neurol. 2017; 8: 124, doi: 10.3389/fneur.2017.00124, indexed in Pubmed: 28421034.

88. Beh SC. External trigeminal nerve stimulation: Potential rescue treatment for acute vestibular migraine. J Neurol Sci. 2020; 408: 116550, doi: 10.1016/j.jns.2019.116550, indexed in Pubmed: 31677559.

89. Beh SC, Friedman DI. Acute vestibular migraine treatment with noninvasive vagus nerve stimulation. Neurology. 2019; 93(18): e1715e1719, doi: 10.1212/WNL.0000000000008388, indexed in Pubmed: 31554650.

90. Kong WJ, Scholtz AW, Kammen-Jolly K, et al. Ultrastructural evaluation of calcitonin gene-related peptide immunoreactivity in the human cochlea and vestibular endorgans. Eur J Neurosci. 2002; 15(3): 487-497, doi: 10.1046/j.0953-816x.2001.01880.x, indexed in Pubmed: 11876776.

91. Xiaocheng W, Zhaohui S, Junhui X, et al. Expression of calcitonin gene-related peptide in efferent vestibular system and vestibular nucleus in rats with motion sickness. PLoS One. 2012; 7(10): e47308, doi: 10.1371/journal.pone.0047308, indexed in Pubmed: 23056625.

92. Bayer O, Adrion C, Al Tawil A, et al. PROVEMIG investigators. Results and lessons learnt from a randomized controlled trial: prophylactic treatment of vestibular migraine with metoprolol (PROVEMIG). Trials. 2019; 20(1): 813, doi: 10.1186/s13063-019-3903-5, indexed in Pubmed: 31888723.

93. Kaya I, Eraslan S, Tarhan C, et al. Can verapamil be effective in controlling vertigo and headache attacks in vestibular migraine accompanied with Meniere's disease? A preliminary study. J Neurol. 2019; 266(Suppl 1): 62-64, doi: 10.1007/s00415-019-09309-w, indexed in Pubmed: 30989371.

94. Liu F, Ma T, Che X, et al. The Efficacy of Venlafaxine, Flunarizine, and Valproic Acid in the Prophylaxis of Vestibular Migraine. Front Neurol. 2017; 8: 524, doi: 10.3389/fneur.2017.00524, indexed in Pubmed: 29075232.

95. Salmito M, Duarte J, Morganti L, et al. Prophylactic treatment of vestibular migraine. Braz J Otorhinolaryngol. 2017; 83(4): 404-410, doi: 10.1016/i.bjorl.2016.04.022.

96. Çelebisoy N, Gökçay F, Karahan C, et al. Acetazolamide in vestibular migraine prophylaxis: a retrospective study. Eur Arch Otorhinolaryngol. 2016; 273(10): 2947-2951, doi: 10.1007/s00405-015-3874-4, indexed in Pubmed: 26728486.

97. Salviz M, Yuce T, Acar H, et al. Propranolol and venlafaxine for vestibular migraine prophylaxis: A randomized controlled trial. Laryngoscope. 2016; 126(1): 169-174, doi: 10.1002/lary.25445, indexed in Pubmed: 26228645.

98. Cassano D, Pizza V, Busillo V. P074. Almotriptan in the acute treatment of Vestibular migraine: a retrospective study. J Headache Pain. 2015; 16(Suppl 1): A114, doi: 10.1186/1129-2377-16-S1-A114, indexed in Pubmed: 28132274.

99. Teggi R, Colombo B, Gatti O, et al. Fixed combination of cinnarizine and dimenhydrinate in the prophylactic therapy of vestibular migraine: an observational study. Neurol Sci. 2015; 36(10): 1869-1873, doi: 10.1007/s10072-015-2270-6, indexed in Pubmed: 26037548.

100. Taghdiri F, Togha M, Razeghi Jahromi S, et al. Cinnarizine for the prophylaxis of migraine associated vertigo: a retrospective study. Springerplus. 2014; 3: 231, doi: 10.1186/2193-1801-3-231, indexed in Pubmed: 24834377.

101. Mikulec AA, Faraji F, Kinsella $\sqcup$. Evaluation of the efficacy of caffeine cessation, nortriptyline, and topiramate therapy in vestibular migraine and complex dizziness of unknown etiology. Am J Otolaryngol. 2012; 33(1): 121-127, doi: 10.1016/i.amjoto.2011.04.010, indexed in Pubmed: 21704423.

102. Neuhauser H, Radtke A, von Brevern M, et al. Zolmitriptan for treatment of migrainous vertigo: a pilot randomized placebo-controlled trial. Neurology. 2003; 60(5): 882-883, doi: 10.1212/01. wnl.0000049476.40047.a3, indexed in Pubmed: 12629256. 